\title{
Reliable Localization for Wireless Sensor Networks in Complex Environments
}

\author{
Xiaolei Liu ${ }^{1}$, Yongji Ren ${ }^{2 *}$, Xuguang Xin ${ }^{2}$, Liping Zhang ${ }^{2}$, Jixiang Chen ${ }^{2}$ \\ ${ }^{1}$ Department of Electrical Engineering, Yantai Vocational College, Yantai, Shandong \\ 264670, China \\ ${ }^{2}$ Department of Command, Naval Aeronautical and Astronautical University, Yantai \\ 264001, China \\ *lenglengqiuyu@sina.com
}

\begin{abstract}
Although localization has been widely studied for Wireless Sensor Networks (WSNs), the complex environments and the large network scale pose severe challenges and make it necessary to develop new reliable localization algorithms. In this paper, we propose a novel Multi-Hop Localization Algorithm for large-scale WSNs in complex environments. This work is based on the consideration that the localization process would encounter several kinds of adverse factors with different nature at the same time (e.g. anisotropic network characteristics, ranging uncertainty, link quality of multihop paths, etc.), which lead to obvious degradation of localization performance. Unlike most of the existing schemes, we transform the localization problem in complex environments into a hybrid constraint satisfaction problem (CSP) which is composed of three different kinds of constraints, i.e. spatial constraint, network situation constraint, and confidence constraint. Set-membership approach and interval analysis method have been utilized to deal with the CSP and determine the positions of sensor nodes. Simulation results show that our scheme is an effective and efficient approach to localization in large-scale WSNs.
\end{abstract}

Keywords: Wireless Sensor Networks, Multi-Hop Localization, Constraint Satisfaction Problem, Set-Membership Approach

\section{Introduction}

Wireless sensor networks (WSNs) have great potential to provide highly effective and convenient solutions for civilian and military extensive monitoring applications, e.g. target tracking, surveillance, environmental monitoring, etc. One important reason is that WSNs can be rapidly and randomly deployed in a large-scale region by plane or unmanned aerial vehicle (UAV), of which the environments are always complex even inaccessible, and then perform tracking or other tasks without manual intervention.

As we know, knowledge about sensor nodes' geometrical positions is no doubt an indispensable part of sensor readings in most WSNs applications. Thus localization has been considered as a fundamental and essential task in WSNs and received more and more attention in recent years. Extensive research has been conducted in this interesting area and a comprehensive survey is provided in [1-3] and the references therein. Some of them are mainly designed for small-scale networks, although that is also an interesting field, we will not contribute to the region in this paper; instead, we focus on the large-scale localization context. 
According to the practical scenarios, WSNs are usually deployed sparsely in a large-scale monitoring area with a minority of anchor nodes which have GPS receivers (or other localization equipment) to get geometrical positions directly. The ordinary nodes, which need to be localized, generally seek for the locations as accurate as possible through various anchor-based solutions. However, the large network scale and the complex environments pose great challenges for this anchor-based localization approach.

On the one hand, for ordinary nodes, there are not enough anchor nodes which only one hop away to communicate and measure distances directly for localizing. To address this problem, three main kinds of localization algorithms have been presented, namely, mobile anchor assisted localization schemes [4-5], recursive schemes [6-7], and multi-hop localization schemes [8-9], respectively. Hereinto, mobile anchor assisted localization methods commonly employ Autonomous Underwater Vehicle (AUV) or other mobile equipment to assist localization. Recursive schemes adopted upgrading some ordinary nodes that have been localized to be secondary anchor nodes to assist other nodes in estimating locations. Whereas in multi-hop localization algorithms, the ordinary node can infer the distances to its non-neighboring anchor nodes by approximating the length of the shortest path to the Euclidean distance, so as to get enough anchor nodes with known distances for localization. The multi-hop localization algorithms not only have no additional requirements for expensive mobile devices but also can provide better real-time performance. Hence people pay more attention to multi-hop localization and this paper also focuses on it.

On the other hand, for the large-scale WSNs within complex environments, due to various adverse factors such as sparse and nonuniform node distribution, irregular radio propagation pattern, or different kinds of complex terrain condition, the network usually revealed obvious anisotropic characteristics. One example of this coexistence of multiple anisotropic characteristics can be found in Fig.1.

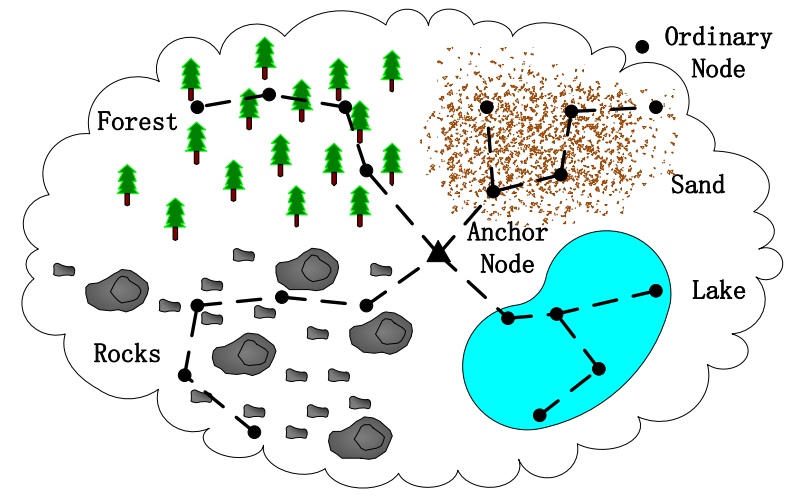

Figure 1 Coexistence of Multiple Anisotropic Patterns

In anisotropic networks, geometric distance between a pair of sensor nodes is not always proportional to their hop count distance, which undermines the accuracy of multi-hop distance estimation. As we know, the accurate distance estimation is the basis of accurate location estimation. However, most of the existing multi-hop schemes are assumed to operate in isotropic networks which consider the hop count distance between two nodes is proportional to their geometric distance. Directly applying these localization schemes proposed for isotropic networks in large-scale anisotropic networks will result in obvious degradation of distance estimation and localization performance. Although a few methods have been proposed for anisotropic networks recently [10-11], they have the inadequacy to focus on only one anisotropic factor (e.g. obstacle detour) and the drawback of nonscalability due to the error accumulation along with the increase of network scale. Obviously, this makes 
it harder for large-scale WSNs to obtain accurate multi-hop distance estimation. Thus this requires us to seek for some effective approach to eliminate the influence of multiple anisotropic factors simultaneously.

Not only that, the whole ranging process also suffers from the low link quality which is mostly due to the complex deployment environments and the time-variability of the medium. Low link quality causes high Bit Error Rate, which further results in degradation of localization performance. There is a need to employ the link quality information in selecting anchor nodes or reference nodes which may improve the accuracy of the distance estimates and consequently improve the location estimates. Moreover, in an adversarial environment, multiple attacks become possible if the malicious anchor nodes may claim fake locations which are different from their physical locations. Consequently, the trust value of each anchor nodes or reference nodes should be considered at the same time.

Therefore, there is a need to develop a novel multi-hop algorithm for producing accurate distance estimation and node coordinates in large-scale and complex WSNs. In this paper, we transform the localization problem in complex environments into a hybrid constraint satisfaction problem (CSP) which is composed of three different kinds of constraints, i.e. spatial constraint, network situation constraint, and confidence constraint. Set-membership approach and interval analysis method have been utilized to deal with the CSP and determine the positions of sensor nodes.

The rest of this paper is organized as follows. In section 2, we describe the novel localization algorithm. Simulation setting and result analysis are given in section 3. And finally, we drew conclusions in section 4.

\section{Reliable Localization Algorithm}

\subsection{Network Initialization}

In this phase, the network will be initialized in a controlled flood manner. For the neighboring nodes, all messages can be exchanged within one hop distance. At the beginning, each node owns a list contains the information of \{ID, type of node, coordinates, distance, hops, type of neighbors $\}$. Through a controlled flood manner, every node can get the related information of their neighbors such as the type of neighbor nodes, distances between neighbor nodes and itself, neighbor surface buoys' coordinates, node local density, etc. Generally, due to the sparse feature of deployment, few nodes can get three or more neighboring surface buoys. Therefore, it is necessary to make a further messages exchange. Based on the analysis of neighboring nodes information, node $N_{p}$, for example, can get to know whether its neighbor node $N_{q}$ owns enough nodes with known locations and decide whether or not to send a message about one-hop anchor nodes to $N_{q}$. If there are still any nodes which are lack of enough nodes with coordinates, they can select some three-hop anchors which owned better environment by analyzing the two-hop information. At the end, all nodes can get their related information such as distances to neighbors, information of neighbor nodes, multi-hop density, multi-hop count, etc.

At the same time, an extension of Self-adaption Link-quality Detection Algorithm (SLDA) [12] is integrated into the initialization phase to detect the link quality of the paths to neighboring anchor nodes or multi-hop anchors. This method adopts Self-adaptive Linkquality Detection strategy to measure the real-time link quality weight factor (LQWF), and combines energy consumption model of mobile nodes to predict optimal transmission path for 
message forwarding. On this basis, the link quality information will be employed to assist in selecting optimal anchor nodes or reference nodes which may improve the accuracy of the distance estimates and consequently improve the location estimates. This phase is performed as follows: Mobile nodes execute a real-time detection to the communication links with a method called communication time slot probing. After this they can acquire adaptive quality factor of the links. This factor dynamically changes with the network's link quality, so it can reflect a real-time feature of varying network situation; Secondly nodes will establish an optimal link decision function through link quality factor and their energy consumption model. According to the value of the function, nodes can select the optimal single route for forwarding.

\subsection{Set-membership Localization Framework}

In this paper, we present a set-membership localization framework, which is deployed at each ordinary node to estimate its location. The design of this framework is inspired by the fact that from the perspective of whole distance measurements uncertainty caused by ambient noise and anisotropy, although we cannot obtain the exact distribution, it is reasonable and natural to assume that the uncertainty is bounded in a certain interval in most practical scenarios.

Depending on the hypothesis on the noise, estimation methods can be classified into statistical estimation and set-membership estimation. In statistical estimation the noise is always represented as a stochastic process, while in set-membership estimation the noise is supposed to be unknown but bounded, with known bound, i.e. UBB noise. As a well-known estimation approach, set-membership only needs the error bound as prior condition; it requires no statistical assumptions on various disturbances. Its aim is to determine a feasible solution set which contains all admissible solutions. The feasible set should be compatible with all the available information such as the UBB noise, the observed measurements, etc. This approach has been shown to be efficient, robust and reliable in the bounded-error context [13]. Thus, it is safe for us to solve the localization issues in set-membership framework.

Initially, consider a situation in which the three dimensional WSN consists of $P$ anchor nodes with known positions and $Q$ ordinary nodes which need to be localized. Let $W_{i}=\left[x_{i}, y_{i}, z_{i}\right]^{T}$ denote the location of node $X_{i}$. The measured distance from ordinary node $X_{u}$ to anchor node $X_{i}$ can be defined as $d_{u i}=D_{u i}+e_{u i}$, where $D_{u i}=\left\|\boldsymbol{W}_{u}-\boldsymbol{W}_{i}\right\|_{2}$ is the Euclidean distance, $e_{u i}$ is considered as the unknown but bounded noise and assumed to be bounded in a certain interval $\left|e_{u i}\right|<c$ with bound value $c$, which accounts for all the uncertainties, e.g. the ambient noise, multi-hop errors, etc. If $X_{i}$ is a multi-hop anchor node of $X_{u}$, then the measured distance $d_{u i}$ can be inferred through multi-hop information exchange in the same manner as conventional multi-hop distance estimation approach. But the advantage of our framework is that both the uncertainty in direct ranging process and the uncertainty caused by multi-hop process in anisotropic network can be treated as additional uncertainty and casted into UBB context. Then, the localization issues can be tackled in the set-membership framework. 


\subsection{Feasible Set and Constraint Problem}

According to the set-membership estimation theory, the localization problem can be described as following:

We wish to estimate the location $W_{u}=\left[x_{u}, y_{u}, z_{u}\right]^{T}$ of ordinary node $X_{u}$ on that basis of measures of the function

$$
F(w)=\left\|\boldsymbol{W}_{u}-\boldsymbol{W}_{i}\right\|_{2}
$$

corrupted by additive noise $e_{u i}$, bounded in a certain interval $\left|e_{u i}\right|<c_{u i}$, for some constant $c$. In such a case, the desired position estimate is guaranteed to be bounded by an admissible space known as feasible set:

$$
\boldsymbol{F}(\boldsymbol{w})=\bigcap_{i=1}^{k}\left\{w \in R^{3}: d_{u i}-c_{u i} \leq\left\|\boldsymbol{W}_{u}-\boldsymbol{W}_{i}\right\|_{2} \leq d_{u i}+c_{u i}\right\}
$$

where $\boldsymbol{W}_{i}$ is the position of the $i$ th anchor node with measured distance to $X_{u}$, $i=1, \cdots, k$. If $X_{i}$ is a one-hop anchor node of $X_{u}$, then $d_{u i}$ amounts to the sample mean $\hat{\theta}_{u i}$ with stand deviation $\hat{\sigma}_{u i}$. If $X_{i}$ is a multi-hop anchor node, then the lower bound of the multi-hop distance estimation becomes the communication radius $R$ when it is smaller than $R$.

By analysing the geometric features, we can draw the following conclusions: (i) the feasible set $\boldsymbol{F}(\boldsymbol{w})$ is actually the intersection of all the spherical caps of center $\boldsymbol{W}_{i}$ and of internal and external radii $r_{u i}=d_{u i}-c_{u i}$ and $R_{u i}=d_{u i}+c_{u i}$, respectively; (ii) $\boldsymbol{F}(\boldsymbol{w})$ may have a complicated geometrical shape, the different thickness of each spherical cap depends on the bound value $c_{u i}$.

Clearly, the complicated geometrical shape of $\boldsymbol{F}(\boldsymbol{w})$ makes the exact computation infeasible due to the limited computational ability of ordinary nodes. To solve this problem, each spherical cap has been considered as a constraint of spatial position in this paper, and then the counting process of feasible set can be formulated as a simple constraint satisfaction problem (CSP).

\subsection{CSP and Location Estimation}

A simple Constraint Satisfaction Problem (CSP), i.e. the determination problem of feasible set, is defined by a set of $k$ constraints, $f_{1}, f_{2}, \cdots, f_{k}$. Each constraint is actually a discriminant relation $f_{i}: d_{u i}-c_{u i} \leq\left\|W_{u}-W_{i}\right\|_{2} \leq d_{u i}+c_{u i}$ linking the corresponding anchor node coordinates as well as the ordinary node. Tools of interval analysis are used in this subsection in order to solve the CSP.

The interval analysis represents a rigorous active field in scientific computation. This growing branch of applied mathematics aims to manipulate intervals instead of real numbers. The interval analysis approach treats intervals as a new kind of numbers represented by the ordered pair of its endpoints. The distance from $X_{u}$ to $X_{i}$ $\left[d_{u i}-c_{u i}, d_{u i}+c_{u i}\right]$ can be denoted by $\zeta_{u 1}^{l}=\left[\zeta_{u 1}^{l-}, \zeta_{u 1}^{l+}\right]$, where $\zeta_{u 1}^{l-}$ and $\zeta_{u 1}^{l+}$ are the corresponding minimal and maximal bounds of $\zeta_{u 1}^{l}$, respectively. The set of the intervals regarding to $X_{u}$ actually is the set of the constraints $f_{1}, f_{2}, \cdots, f_{k}$. Solving the CSP 
problem in an interval analysis framework consists of finding the intersection that contains all possible solutions.

Set theory operations can be applied to intervals counting. Consider two intervals $\zeta_{u 1}^{l}$ and $\zeta_{u 2}^{l}$, their intersection is always an interval which can be denoted as:

$$
\zeta_{u 1}^{I} \cap \zeta_{u 2}^{I}=\left[\left\{\zeta \mid \zeta \in \zeta_{u 1}^{I}, \zeta \in \zeta_{u 2}^{I}\right\}\right]
$$

The intersection can be computed by $\zeta_{u 1}^{I} \cap \zeta_{u 2}^{I}=\left[\max \left\{\zeta_{u 1}^{I-}, \zeta_{u 2}^{I-}\right\}, \min \left\{\zeta_{u 1}^{I+} \zeta_{u 2}^{I+}\right\}\right]$

Therefore, the $\boldsymbol{F}(\boldsymbol{x})$ of ordinary node $X_{u}$ can be rewritten as:

$$
F_{u}=\bigcap_{i=1}^{k}\left\{\zeta \in \zeta^{I}: \zeta_{u i}^{l}=\left[\zeta_{u i}^{I-}, \zeta_{u i}^{I+}\right]\right\}
$$

Regarding the coordinates of all sub-boxes' centers as samples of $X_{u}$, we can get a sample set $F_{u}=\left\{\Theta_{1}, \Theta_{2}, \cdots, \Theta_{n}\right\}$, and the centre of $\Theta_{n}$ can be found by $\zeta_{n}^{*}=\left(\zeta_{n}^{-}+\zeta_{n}^{+}\right) / 2$. Then the optimum point estimate, i.e. the desired coordinates of ordinary node $X_{u}$, can be obtained by

$$
\begin{gathered}
\hat{\boldsymbol{W}}_{u}=\underset{W_{u}}{\arg \min } \sum_{i=1}^{k}\left(\left\|\zeta_{n}^{*}-\boldsymbol{W}_{i}\right\|_{2}-d_{u i}\right)^{2} \\
\text { subject to } W_{u} \in F(w)
\end{gathered}
$$

\section{Performance Evaluation}

In this paper, we conduct extensive simulations to compare the performance of our scheme with conventional method DV-distance by employing localization error. The localization error is defined as the ratio of Euclidean distance between estimated position and the real position to communication radius $\mathrm{R}$. In our simulation, 300 sensor nodes ( $10 \%$ anchors) with adjustable transmission range $\mathrm{R}$ are randomly distributed in an $\mathrm{H}$ shaped area within a $(3000 \mathrm{~m}, 3000 \mathrm{~m}, 20 \mathrm{~m})$ volume to form an anisotropic network (see Fig. 2).

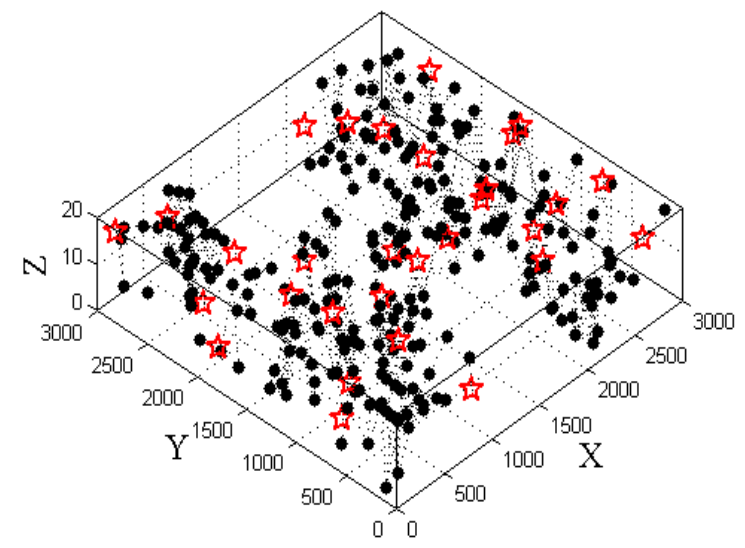

Figure 2 Topology of the Anisotropic Wireless Sensor Networks (Hshape)

For comparison with the classical approaches, measurement noise has been assumed to follow the uniform distribution within the interval $[-100 \mathrm{~m} 100 \mathrm{~m}]$ and the normal distribution with mean value and standard deviation $(10 \mathrm{~m}, 10 \mathrm{~m})$, respectively. In both cases, our 
algorithm assumes the knowledge of the error bound is $30 \mathrm{~m}$. The default parameters are shown in Table 1. To reduce the influence of outliers, we run each simulation 100 times and take the average results as the final data points. Unless specified, we use the default parameters in the following simulations.

Table 1 Default Network Configuration Parameters

\begin{tabular}{|l|l|}
\hline Parameters & Values \\
\hline Network size & $3000 \mathrm{~m} \times 3000 \mathrm{~m} \times 20 \mathrm{~m}$ \\
Deployment strategy & H-shape anisotropic networks \\
Number of sensor nodes & 300 \\
Number of anchor nodes & 30 \\
Number of ordinary nodes & 270 \\
Number of simulation rounds & 100 \\
TTL & 4 \\
Communication radius $(R)$ & $190 \mathrm{~m}$ (adjustable) \\
the standard deviation & 10 \\
Network connectivity & $4 \sim 15$ \\
\hline
\end{tabular}

Fig. 3 presents the distribution boxplots of node localization errors which are normalized by $R$. The y-axis is drawn in log-scale. Box 1 and 3 are the distribution boxplots of DVdistance approach followed normal and uniform distribution, respectively. Box 2 and 4 are the boxplots of our approach which followed normal and uniform distribution. We can see that the average errors and median errors of our scheme are much smaller than those of DVdistance in different noisy environment. In our scheme, the localization errors of almost all nodes can be controlled below $15 \%$. Clearly, our scheme can get better performance for most nodes, which is especially important for large-scale WSNs. It indicates that our scheme is efficient and robust to hybrid noise-processing in anisotropic WSNs localization.

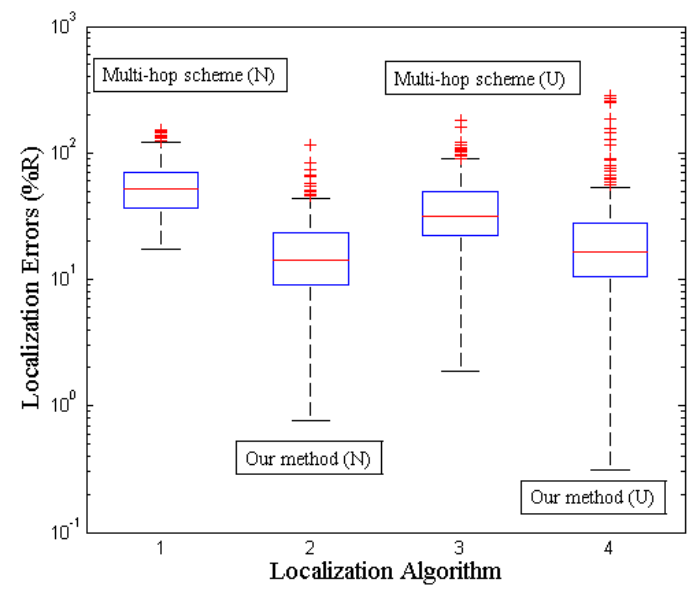

Figure 3 Distribution Boxplots of Node Localization Errors

Next, we vary the communication radius and get the accuracy comparisons under different network connectivity, ranging from 4 to 15 . Fig. 4 shows the average localization errors with the changing network connectivity. It can be seen that our scheme outperforms DV-distance in both uniform distribution and normal distribution cases. We can also see that our method is less affected by the variation of distributions. Particularly in large-scale and sparse network with smaller connectivity and anchor percentage, our method can get higher average localization accuracy. 


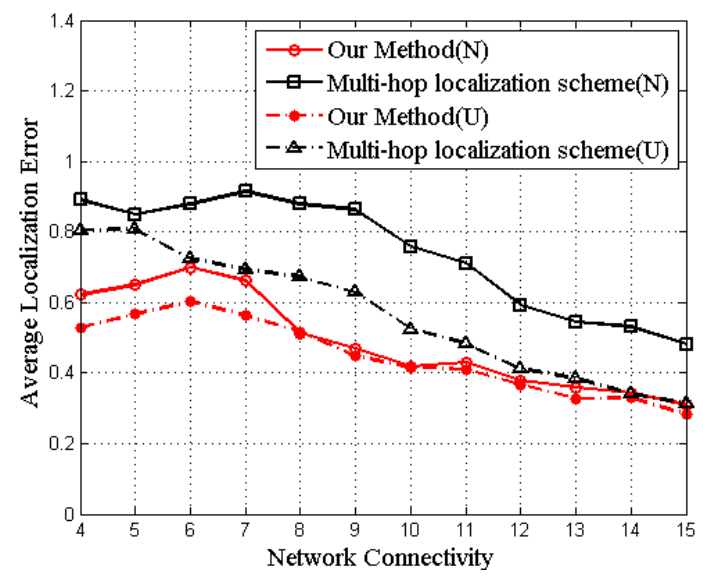

Figure 4 Average Localization Errors Versus Network Connectivity

\section{Conclusion}

In this paper, we propose a novel Multi-Hop Localization Algorithm for large-scale WSNs in complex environments. This work is based on the consideration that the localization process would encounter several kinds of adverse factors with different nature at the same time, which leads to obvious degradation of localization performance. Unlike most of the existing schemes, we transform the localization problem in complex environments into a hybrid CSP which is composed of three different kinds of constraints, i.e. spatial constraint, network situation constraint, and confidence constraint. Set-membership approach and interval analysis method have been utilized to deal with the CSP and determine the positions of sensor nodes. Simulation results show that our scheme is an effective and efficient approach to localization in large-scale WSNs.

\section{References}

[1] I. F. Akyildiz, W. Su, Y. Sankarasubramaniam and E. Cayirci, "Wireless Sensor Networks: A Survey", Computer Networks, vol. 38, no. 4, (2002), pp. 393-422.

[2] J. Yick, B. Mukherjee, D. Ghosal, "Wireless Sensor Network Survey", Computer Networks, vol. 52, no. 12, (2008), pp. 2292-2330.

[3] C. Buratti, A. Conti, D. Dardari, R. Verdone, "An Overview on Wireless Sensor Networks Technology and Evolution”, Sensors, vol. 9, no. 9, (2009), pp. 6869-6896.

[4] O. Chia-Ho, "A Localization Scheme for Wireless Sensor Networks Using Mobile Anchors with Directional Antennas”, IEEE Sensors Journal, vol. 11, no. 7, (2011), pp. 1607-1616.

[5] M. Erol, L. F. M. Vieira and M. Gerla, “AUV-Aided Localization for Underwater Sensor Networks”, In Proceedings of the Wireless Algorithms, Systems and Applications, (2007), pp. 44-54.

[6] J. Liu and Y. Zhang, "Error Control in Distributed Node Self-Localization", Eurasip Journal on Advances in Signal Processing, vol. 2008, (2008).

[7] K. Yu, Y. J. Guo and M. Hedley, "TOA-Based Distributed Localisation with Unknown Internal Delays and Clock Frequency Offsets in Wireless Sensor Networks”, IET Signal Processing, vol. 3, no. 2, (2009), pp. 106-118.

[8] D. Niculescu and B. Nath, "DV Based Positioning in Ad Hoc Networks", Telecommunication Systems, vol. 22, no. 1-4, (2003), pp. 267-280.

[9] R. J. Feng, X. L. Guo, J. W. Wan, Y. F. Wu and N. Yu, "Multihop Localisation with Distance Estimation Bias for 3D Wireless Sensor Networks", Electronics Letters, vol. 48, no. 14, (2012), pp. 884-885.

[10] H. Lim and J. C. Hou, "Localization for Anisotropic Sensor Networks", In Proceedings of the IEEE INFOCOM 2005, March 13, 2005 - March 17, (2005), pp. 138-149.

[11] C. Wang and L. Xiao, "Sensor Localization in Concave Environments", ACM Transactions on Sensor Networks, vol. 4, no. 31, (2008). 
[12] X. Wang, Y. Wu, N. Yu and J. Wan, "A Self-Adaption Link-Quality Detection Algorithm for Data Collecting in OSN", in Services Computing Conference (APSCC), 2010 IEEE Asia-Pacific, (2010), pp. 516-522.

[13] M. Milanese and A. Vicino, "Optimal estimation theory for dynamic systems with set membership uncertainty, An overview”, Automatica, vol. 27, no. 6, (1991), pp. 997-1009.

\section{Authors}

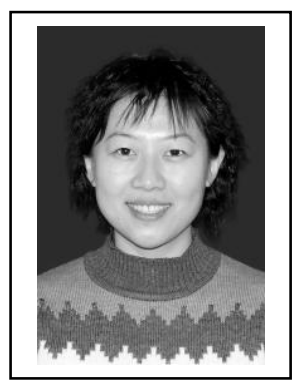

\section{Xiaolei Liu}

Xiaolei Liu is a teacher in Yantai Vocational College, China. She received the B.S., M.S. degrees from Shandong University in 2003 and 2006, respectively. Her research interests include Wireless Sensor Networks, Underwater Wireless Sensor Networks, Localization and Tracking, Network Security and Information Fusion.

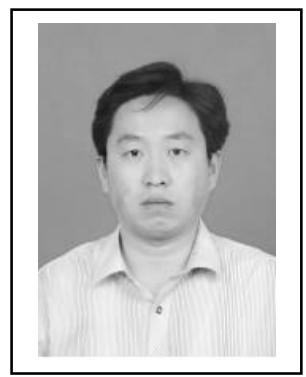

\section{Yongji Ren}

Yongji REN is a Ph.D. student in School of Instrumentation Science and Opto-electronics Engineering at Beijing University of Aeronautics and Astronautics, China. He received the B.S. degree from Shandong University in 2003. His research interests include Wireless Sensor Networks, Underwater Wireless Sensor Networks, Localization and Tracking, Network Security and Information Fusion. 
International Journal of Control and Automation Vol.7, No.10 (2014) 